\title{
Aging and rejuvenation with fractional derivatives
}

\author{
Gerardo Aquino, ${ }^{1}$ Mauro Bologna, ${ }^{1}$ Paolo Grigolini, ${ }^{1,2,3}$ and Bruce J. West ${ }^{4}$ \\ ${ }^{1}$ Center for Nonlinear Science, University of North Texas, P. O. Box 311427, Denton, Texas 76203-1427, USA \\ ${ }^{2}$ Dipartimento di Fisica dell'Università di Pisa and INFM, via Buonarroti 2, 56127 Pisa, Italy \\ ${ }^{3}$ Istituto dei Processi Chimico Fisici del CNR, Area della Ricerca di Pisa, Via G. Moruzzi 1, 56124 Pisa, Italy \\ ${ }^{4}$ Mathematics Division, Army Research Office, Research Triangle Park, North Carolina 27709, USA
}

(Received 18 March 2004; published 10 September 2004)

\begin{abstract}
We discuss a dynamic procedure that makes fractional derivatives emerge in the time asymptotic limit of non-Poisson processes. We find that two-state fluctuations, with an inverse power-law distribution of waiting times, finite first moment, and divergent second moment, namely, with the power index $\mu$ in the interval $2<\mu<3$, yield a generalized master equation equivalent to the sum of an ordinary Markov contribution and a fractional derivative term. We show that the order of the fractional derivative depends on the age of the process under study. If the system is infinitely old, the order of the fractional derivative, $a$, is given by $a=3$ $-\mu$. A brand new system is characterized by the degree $a=\mu-2$. If the system is prepared at time $-t_{a}<0$ and the observation begins at time $t=0$, we derive the following scenario. For times $0<t \ll t_{a}$ the system is satisfactorily described by the fractional derivative with $o=3-\mu$. Upon time increase the system undergoes a rejuvenation process that in the time limit $t \gg t_{a}$ yields $a=\mu-2$. The intermediate time regime is probably incompatible with a picture based on fractional derivatives, or, at least, with a mono-order fractional derivative.
\end{abstract}

DOI: 10.1103/PhysRevE.70.036105

PACS number(s): 02.50.Ey, 05.40.Fb, 05.60.Cd

\section{INTRODUCTION}

The fractional calculus has recently received a great deal of attention in the physics literature, through the publication of books [1,2] and review articles [3,4], as well as an ever increasing number of research papers, some of which are quoted here [5-12]. The blossoming interest in the fractional calculus is due, in part, to the fact that it provides one of the dynamical foundations for fractal stochastic processes [2,4]. The adoption of the fractional calculus by the physics community was inhibited historically because there was no clear experimental evidence for its need. The disciplines of statistical physics and thermodynamics were thought to be sufficient for describing complex physical phenomena solely with the use and modifications of analytic functions. This view was supported by the successes of such physicists as Onsager, who through the use of simple physical arguments was able to relate apparently independent transport processes to one another, even though these processes are associated with quite different physical phenomena [14]. His general arguments rested on three assumptions: (1) microscopic dynamics have time-reversal symmetry; (2) fluctuations of the heat bath decay at the same rate as do macroscopic deviations from equilibrium; and (3) physical systems are aged. We refer to assumption 2 as the Onsager principle and show that it is tied up with assumption 3.

Onsager's arguments focused on a system that is in contact with a heat bath sufficiently long that the bath has come to thermal equilibrium and consequently the system is aged. In statistical physics we know that the bath is responsible for both fluctuations and dissipation, and if the fluctuations are white the regression of perturbations of the bath to equilibrium is instantaneous. This means that the energy absorbed from the system of interest by the bath, through macroscopic dissipation, is distributed over the bath degrees of freedom on a very much shorter time scale than the relaxation time of the system. This property is summarized in the well known fluctuation-dissipation theorem, which has even been generalized to the case where the fluctuations in the bath do not regress instantaneously [15].

The dynamics of the physical variables to which the Onsager principle apply are described by two different kinds of equations: (1) the Langevin equation, a stochastic differential equation for the dynamical variable and (2) the phase space equation for the probability density. Two distinct methods have been developed to describe the phase space evolution of the probability density: the master equation introduced by Pauli and the continuous time random walk (CTRW) approach of Montroll and Weiss [16]. The CTRW formalism describes a random walk in which the walker pauses after each jump for a sojourn specified by a waiting time distribution function. It was shown by Bedeaux et al. [17] that the Markov master equation is equivalent to a CTRW if the waiting time distribution is Poissonian. However, when the waiting time distribution is not exponential, the case we consider here, the equivalence between the two approaches is maintained only by generalizing to the non-Markov master equation, the so-called generalized master equation (GME) [18]. Recently, Metzler [19] argued that the GME unifies the fractional calculus and the CTRW.

Allegrini et al. [13] have shown that creating a master equation compatible with the Onsager principle requires that the system be entangled with the bath in such a way as to realize a condition of stable thermodynamic equilibrium. This system-bath entanglement is the result of a rearrangement process that may take an infinitely long time to complete, leading to the replacement of the GME of Kenkre et al. [18], which corresponds to the nonstationary condition, with a new GME compatible with the stationary condition, and consequently with the Onsager principle. Herein, we extend that discussion to include the connection with the fractional calculus in both infinitely and not infinitely aged condition. 
This will allow us to go beyond the discussion of Sokolov and Metzler [12], whose fractional derivative refers to the young state. We shall find a fractional derivative operator with a different index, corresponding to the infinitely aged condition, and we shall show that the condition of Sokolov and Metzler is an attractor for all the systems that are partially aged and not infinitely aged.

\section{Beyond the Onsager principle}

We approach the subject of fractional derivatives from a perspective similar to that of Sokolov and Metzler [12]. More specifically, we address the problem of the connection between the GME [18] and the stationary version of the CTRW [16]. The GME considered by Allegrini et al. [13] is the two-site version of the following equation:

$$
\frac{d}{d t} \mathbf{p}(t)=-\int_{0}^{t} \Phi\left(t-t^{\prime}\right) \mathbf{K} \mathbf{p}\left(t^{\prime}\right) d t^{\prime},
$$

where $\mathbf{p}(t)$ is the $m$-dimensional population vector of $m$ sites, $\mathbf{K}$ is a transition matrix between the sites, and $\Phi(t)$ is the memory kernel. The CTRW prescription for this process yields

$$
\mathbf{p}(t)=\sum_{n=0}^{\infty} \int_{0}^{t} d t^{\prime} \psi_{n}\left(t^{\prime}\right) \Psi\left(t-t^{\prime}\right) \mathbf{M}^{n} \mathbf{p}(0) .
$$

Note that $\psi_{n}(t)$ is the probability that $n$ jumps occurred and that the last jump took place at time $t=t^{\prime}$, implying the renewal theory relation

$$
\psi_{n}(t)=\int_{0}^{t} \psi_{n-1}\left(t-t^{\prime}\right) \psi_{1}\left(t^{\prime}\right) d t^{\prime},
$$

where $\psi_{1}(t)$ is the waiting time distribution function $\psi(t)$ introduced into the CTRW and $\psi_{0}(t)=\delta(t)$. While $\mathbf{M}$ is the transition matrix connecting the sites after one jump has occurred, the probability that no jump occurs in the time interval $(0, t)$ is

$$
\Psi(t)=\int_{t}^{\infty} \psi\left(t^{\prime}\right) d t^{\prime}
$$

The waiting time distribution function and the memory kernel can be related to one another by taking the Laplace transform of the GME (1) and the CTRW (2). This comparison, after some algebra [13], yields

$$
\hat{\Phi}(u) \mathbf{K}=\frac{u \hat{\psi}(u)}{1-\hat{\psi}(u)}(\mathbf{M}-\mathbf{I}),
$$

where $\mathbf{I}$ is the $m \times m$ unit matrix and the Laplace transform of the function $f(t)$ is $\hat{f}(u)$. Here, as in Allegrini et al. [13], we limit our discussion to the two-state case where

$$
\mathbf{M}=\left(\begin{array}{ll}
0 & 1 \\
1 & 0
\end{array}\right)
$$

and

$$
\mathbf{K}=\left(\begin{array}{cc}
1 & -1 \\
-1 & 1
\end{array}\right),
$$

thereby reducing Eq. (5) to

$$
\hat{\Phi}(u)=\frac{u \hat{\psi}(u)}{1-\hat{\psi}(u)} .
$$

This relation between the Laplace transform of the memory kernel and the Laplace transform of the waiting time distribution function was first obtained by Kenkre et al. [18] and is reviewed by Montroll and West [20].

In the case when the lattice has only two sites, a left and a right site, the random walker corresponds to a dichotomous signal $\xi$, with the values $\xi(t)=-1$, for the left site, and $\xi(t)$ $=1$, for the right site. For the sake of simplicity, we assume the two states to have the same statistical weight. Also in the two-state CTRW, if we adopt a discrete time representation, the motion of the random walker corresponds to a symbolic sequence $\{\xi\}$, with the form $\{+++++++-+--++++---$ --_- $\cdots\}$, which shows a significant persistence of both states. The waiting time distribution $\psi(t)$ is the distribution of the patches filled with either +'s or -'s.

We assume symmetry between the two states and a finite first moment of $\psi(t)$ making it possible for us to define the autocorrelation function for the fluctuations $\xi(t)$,

$$
\Phi_{\xi}(t)=\frac{\langle\xi(0) \xi(t)\rangle}{\left\langle\xi^{2}\right\rangle},
$$

because the process is stationary in time [21]. The problem of how to relate $\Phi_{\xi}(t)$ to this persistent fluctuation is delicate and will be discussed with some detail in Sec. I B. Here we limit ourselves to noticing that the Onsager principle bypasses the technical difficulties with the connection between the Langevin and the master equation pictures, either ordinary or generalized. In fact, the master equation is a prescription to determine the probability of occupying a given state. In the case under study here, the master equation makes it possible to determine $p_{i}(t)$, at time $t$, with $i=1,2$. The Langevin equation, or in general any theoretical tool driving the motion of a variable, $\xi(t)$ in the case under study here, affords a convenient means to determine $\xi(t)$, and consequently the autocorrelation function of Eq. (9).

In his original work Onsager considered the case of a macroscopic fluctuation that regresses to equilibrium through the phenomenological equations of motion. Here we adopt an extension of the Onsager principle made by Allegrini $e t$ $a l$. [13] to the case of two states, using the probability of the random walker being in state $i=1,2, p_{i}(t)$, at time $t$, which allows us to determine the autocorrelation function of Eq. (9) without any need to establish the Langevin-like picture equivalent to the GME under study here. In fact, we adopt the following equality:

$$
\Phi_{\xi}(t)=\frac{p_{1}(t)-p_{2}(t)}{p_{1}(0)-p_{2}(0)},
$$

which yields an immediate connection between the GME of Eq. (1) and the autocorrelation function of Eq. (9), provided 
that the system is initially in the out of equilibrium state corresponding to $p_{1}(0)-p_{2}(0) \neq 0$. Assuming a regression to equilibrium in such a way as to retain Eq. (10) we obtain from the GME (1) using the coupling matrix (7)

$$
\frac{d \Phi_{\xi}(t)}{d t}=-2 \int_{0}^{t} \Phi\left(t-t^{\prime}\right) \Phi_{\xi}\left(t^{\prime}\right) d t^{\prime} .
$$

Thus, the Laplace transform of the autocorrelation function can be related to the Laplace transform of the memory kernel by

$$
\hat{\Phi}_{\xi}(u)=\frac{1}{u+2 \hat{\Phi}(u)} .
$$

It is interesting to notice that in the Poisson case, namely, when $\psi(t)$ is an exponential function of time, the memory kernel of the GME, given by Eq. (8) turns out to be equivalent to a Dirac $\delta$ function of time, thereby implying that the bath responsible for the fluctuations of the variable $\xi$ has a time scale infinitely smaller than the system of interest. In this specific case, with the help of Eq. (12) we see that the autocorrelation function $\Phi_{\xi}(t)$ decays exponentially with time. This is a condition behind the ordinary Onsager principle. Following the authors of Ref. [13] we want to go beyond the ordinary Onsager principle.

The authors of Ref. [13] studied the case where the autocorrelation function departs from the exponential relaxation and has the the following time asymptotic property:

$$
\Phi_{\xi}(t)_{t \rightarrow \infty} \sim \frac{1}{t^{\mu-2}} .
$$

The waiting time distribution $\psi(t)$ corresponding to this autocorrelation function has the following time asymptotic property [13]:

$$
\psi(t)_{t \rightarrow \infty} \sim \frac{1}{t^{\mu}},
$$

with $\mu>2$ to fit the stationary condition. At first sight, one might be surprised about our decision to make these complex processes obey the Onsager principle. Such processes have exotic thermodynamical properties, and in some cases they are even shown to be nonergodic [22] and to produce aging effects $[23,26]$. Another interesting effect emerging from these processes was described in Ref. [24]. These authors used a fractional Fokker-Planck equation, which is closely related to the GME used in this paper, and they found that the response of their GME to external perturbation is quite different from the response of the corresponding CTRW, in conflict with the fact that their GME is equivalent to a CTRW in the absence of perturbation. All these surprising properties, however, refer to the case $\mu<2$, where no invariant measure exists. The case $\mu>2$, under study here, is in principle compatible with the Onsager principle, and as a consequence our request is not absurd. Nevertheless, we shall see that the Onsager principle requires that the system is infinitely aged, an ideal condition, and that an even apparently negligible departure from this condition yields a striking effect: a rejuvenation process.
The authors of Ref. [13] determined that the problem of how to make these processes compatible with the Onsager principle could be solved by expressing the CTRW in stationary form, resulting in the GME memory kernel

$$
\hat{\Phi}(u)=\frac{u[1-\hat{\psi}(u)]}{-2[1-\hat{\psi}(u)]+u[1+\hat{\psi}(u)] \tau},
$$

where $\tau$ is the average waiting time,

$$
\tau=\int_{0}^{\infty} t \psi(t) d t
$$

The form of the memory kernel given by Eq. (15) is consistent with the equation of motion for the autocorrelation function (11), and consequently Eq. (15) is equivalent to

$$
\hat{\Phi}(u)=\frac{1}{2}\left(\frac{1}{\hat{\Phi}_{\xi}(u)}-u\right) .
$$

\section{B. Theoretical waiting time distribution}

We have to remind the reader that the stationary autocorrelation function of $\xi$ is not related directly to $\psi(t)$. Zumofen and Klafter [25] provided a prescription for deriving the corresponding equilibrium autocorrelation function of $\xi$ from $\psi(t)$. Their result rests on the observation that $\psi(t)$ is an experimental function, evaluated by observing the time duration of the two states. The connection with renewal theory is established by assuming that the time duration of a state is determined by two processes; one is the extraction of a random number from a theoretical inverse power-law distribution $\psi^{*}(\tau)$, with the same power index $\mu$, and the other is a coin tossing procedure that determines the sign of this laminar region. Thus, a given experimental sojourn time in one of the two states may correspond to an arbitrarily large number of drawings and coin tossings. Renewal theory is used to relate the autocorrelation function $\Phi_{\xi}(t)$ to the waiting time distribution function $\psi^{*}(t)$. In fact, from the renewal theory [21] we obtain the following important result:

$$
\Phi_{\xi}(t)=\frac{1}{\tau^{*}} \int_{t}^{\infty}\left(t^{\prime}-t\right) \psi^{*}\left(t^{\prime}\right) d t^{\prime},
$$

where $\tau^{*}$ is the mean waiting time of the $\psi^{*}(t)$-distribution density. It is interesting to notice that this equation implies that the second derivative of the autocorrelation function is proportional to $\psi^{*}(t)$,

$$
\frac{d^{2}}{d t^{2}} \Phi_{\xi}(t)=\frac{\psi^{*}(t)}{\tau^{*}} .
$$

In Sec. II the departure point of our calculations is given by the autocorrelation function $\Phi_{\xi}(t)$ of Eq. (18). In this case is convenient to assign to this equilibrium autocorrelation function a simple analytical form. This is done as follows.

First of all we assign to $\psi^{*}(t)$ the following analytical form: 


$$
\psi^{*}(t)=(\mu-1) \frac{T^{* \mu-1}}{\left(t+T^{*}\right)^{\mu}},
$$

which, as can be proved with the help of Eq. (24), is compatible with $\psi(t)$ getting the time asymptotic form of Eq. (14). This makes it possible for us to write $\tau^{*}$ as follows:

$$
\tau^{*}=\int_{0}^{\infty} t \psi^{*}(t) d t=\frac{T^{*}}{\mu-2} .
$$

With the choice of Eq. (20) for $\psi^{*}(t)$, the autocorrelation function $\Phi_{\xi(t)}$ of Eq. (18) gets the attractive analytical form

$$
\Phi_{\xi}(t)=\left(\frac{T^{*}}{t+T^{*}}\right)^{\beta},
$$

where

$$
\beta \equiv \mu-2 .
$$

Thus, in the case $\mu<3$, the autocorrelation function of the fluctuations is not integrable.

Zumofen and Klafter [25], in addition to explaining with clear physical arguments the connection between $\psi(t)$ and $\psi^{*}(t)$, established that the Laplace transforms of the two functions are related to one other by

$$
\hat{\psi}^{*}(u)=\frac{2 \hat{\psi}(u)}{1+\hat{\psi}(u)} .
$$

This important relation allows us to establish a connection between $\tau$ and $\tau^{*}$, which turns out to be

$$
\tau=2 \tau^{*} .
$$

In conclusion, there are two different waiting time distributions with the time asymptotic behavior of Eq. (14), the experimental waiting time distribution $\psi(t)$ and the theoretical waiting time distribution $\psi^{*}(t)$. The theoretical distribution is necessary to define the autocorrelation function $\Phi_{\xi}(t)$. Thus, a theoretical treatment involving the autocorrelation function will force us to depend on $\psi^{*}(t)$. In this case it is convenient to adopt the analytical form of Eq. (20) for $\psi^{*}(t)$, and Secs. II and III will rest on this choice. In other cases, Sec. IV, Appendix A, and Appendix B, the theoretical treatment will use the experimental waiting time distribution $\psi(t)$. In these cases it is convenient to adopt the analytical form

$$
\psi(t)=(\mu-1) \frac{T^{\mu-1}}{(t+T)^{\mu}} .
$$

However, whatever choice is made, either the analytical form of Eq. (20) for $\psi^{*}(t)$ or the analytical form of Eq. (26) for $\psi(t)$, in both cases, thanks to Eq. (24), the two waiting time distributions maintain the same time asymptotic behavior, with the same $\mu$. So do the two different expression for the equilibrium autocorrelation function, both decaying as Eq. (13). For the main purpose of this paper the time asymptotic behavior is in fact the property that matters.

\section{Purpose of this paper}

The purpose of this paper is to prove that a dichotomous fluctuation $\xi$, with the waiting time distribution of Eq. (14), can be described by a GME that, in turn, is well represented by a fractional derivative operator. The fractional index corresponds to the realization of the Onsager condition, as surprising as this fact might be in the condition $3>\mu>2$, which is so far from the Poisson limit $\mu=\infty$. The Onsager principle is not totally foreign to this anomalous physical condition, thanks to the fact that $\mu>2$ is compatible with the existence of thermodynamical equilibrium. However, the time necessary to reach this thermodynamical condition is infinite, and the system, observed at finite times, no matter how long, shows a surprising rejuvenation effect. Through this rejuvenation effect, the fractional order compatible with the Onsager principle slowly turns into that established by Sokolov and Metzler [12].

The ouline of the paper is as follows. In Sec. II, using the inverse Laplace transform of Eq. (17) we determine the unknown memory kernel $\Phi(t)$, making it possible to discuss how to express the GME in terms of fractional derivatives. The case where $2<\mu<3$ is compared to the recent work of Sokolov and Metzler [12]. We find that the index of the fractional derivative is $3-\mu$, rather than $\mu-2$, as predicted by Sokolov and Metzler. In Sec. III, we prove that this difference in index is due to the fact that we adopt a stationary condition, while Sokolov and Metzler do not. In Sec. IV, we also prove that in the case of a finite, rather than infinite, age our GME makes a transition from the $(3-\mu)$ th to the $(\mu$ $-2)$ th order. The stationary case becomes stable only in the limiting case of infinite age. In Appendix A, the interested reader can find details on how to establish the order of the fractional operator in the GME, in the whole range $1<\mu<3$, when only the brand new condition of Eq. (8) is considered. The accuracy of the analytical expressions that we use in Sec. IV to illustrate the rejuvenation process is discussed in Appendix B.

In conclusion, on the one hand we shed light on the meaning of the work of Allegrini et al. [13], which is proven to be a subordination to a Markov master equation through the stationary distribution of first exit times. On the other hand, we extend the approach to systems of any age and reveal the phenomenon of a continuous time random walk with rejuvenation. To accomplish this dual role we rely heavily on the results recently obtained by Barkai [26] and, to a lesser extent the results of Allegrini et al. [13]. However, this allows us to reveal a dependence of the fractional derivative order on the aging and rejuvenation process, which was not previously identified.

\section{THE INVERSE LAPLACE TRANSFORM OF THE MEMORY KERNEL}

To establish the form of the unknown memory kernel $\Phi(t)$, we make a few preliminary observations. First of all, we note that through Eq. (17) we establish a direct connection with the autocorrelation function $\Phi_{\xi}(t)$ and that this auto-correlation function is, in turn, directly related to the 
waiting time distribution $\psi^{*}(t)$, through Eq. (18). Thus, with no loss of generality for the reasons illustrated in Sec. I it is convenient to refer to $\psi^{*}(t)$ rather than to $\psi(t)$ and consequently, according to the prescriptions illustrated at the end of Sec. I B, to the analytical form of Eq. (20).

For simplicity, we set $T^{*}=1$ throughout this section. Thus, the Laplace transform of the autocorrelation function is [27]

$$
\hat{\Phi}_{\xi}(u)=\frac{\Gamma(1-\beta)}{u^{1-\beta}}\left(e^{u}-E_{\beta-1}^{u}\right),
$$

where $0<\beta<1$, given the fact that we are considering $2<\mu<3$, and $E_{\beta-1}^{u}$ is a generalized exponential function [2]. Thus, $\hat{\Phi}_{\xi}(u)$ diverges as $u \rightarrow 0$ and Eq. (12) yields $\hat{\Phi}(0)=0$. We explore the opposite limit $u \rightarrow \infty$ using Eq. (15), which yields $\hat{\Phi}(u)=1 / \tau=1 / 2 \tau^{*}$. In the time representation, the latter limit is equivalent to $\Phi(t) \approx \delta(t) / 2 \tau^{*}$ for $t \rightarrow 0$. Therefore, we segment the Laplace transform of the GME memory kernel into two parts as follows:

$$
\hat{\Phi}(u)=\frac{1}{2 \tau^{*}}+\hat{\Phi}_{a}(u) .
$$

The first term models the short-time limit, while the second term is responsible for the long-time behavior. In the time representation we have

$$
\Phi(t)=\frac{\delta(t)}{2 \tau^{*}}+\Phi_{a}(t) .
$$

Note that this division of the memory kernel into a whitenoise contribution and a slow term corresponds to a similar partition made by Fuliński [28].

Thus, for the time evolution equation of the autocorrelation function of $\xi(t)$, we derive the following equation:

$$
\frac{d \Phi_{\xi}(t)}{d t}=-\frac{1}{\tau^{*}} \Phi_{\dot{\xi}}(t)-2 \int_{0}^{t} \Phi_{a}\left(t-t^{\prime}\right) \Phi_{\xi}\left(t^{\prime}\right) d t^{\prime} .
$$

Using Eq. (9) and substituting into it the explicit expression of $\tau^{*}$ as a function of $\mu$, after some algebra, we obtain

$$
\begin{aligned}
\left(\frac{T}{t+T}\right)^{\beta} \frac{\beta t}{T(t+T)}= & \Phi_{\xi}(t) \frac{\beta t}{T(t+T)}=-2 \int_{0}^{t} \Phi_{a}(t \\
& \left.-t^{\prime}\right) \Phi_{\xi}\left(t^{\prime}\right) d t^{\prime} .
\end{aligned}
$$

The two terms on the left-hand side Eq. (31) are positive. Due to the negative sign on the right-hand side of this equation we conclude that it might well be that $\Phi_{a}(t)$ is always negative.

Let us concentrate on the case $\beta<1$ : using the autocorrelation function $\Phi_{\xi}(t)$ of Eq. (22) (with $T^{*}=1$ ) and using the change of time variable $t^{\prime}+1 \rightarrow t^{\prime}$, we rewrite Eq. (31) in the form

$$
\begin{aligned}
& -2 \int_{0}^{t+1} \Phi_{a}\left(t+1-t^{\prime}\right) \frac{1}{t^{\prime \beta}} d t^{\prime}+2 \int_{0}^{1} \Phi_{a}\left(t+1-t^{\prime}\right) \frac{1}{t^{\prime} \beta} d t^{\prime} \\
& =\frac{\beta t}{(t+1)^{\beta+1}} .
\end{aligned}
$$

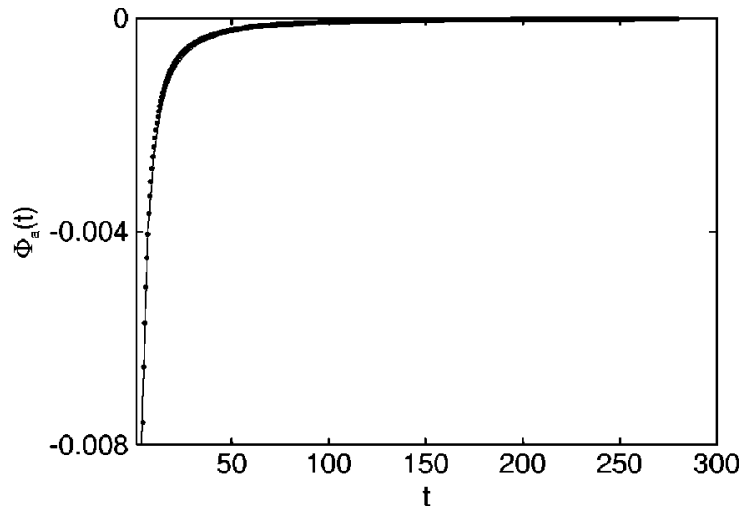

FIG. 1. The slow component of the memory kernel $\Phi(t), \Phi_{a}(t)$, as a function of time. The black dots denote the result of the numerical inversion of the expression in the Laplace transform resulting from Eqs. (15) and (28) for $\beta=0.5$; the continuous line is the analytical approximation given by Eq. (36).

In the limiting case $t \rightarrow \infty$ we neglect the second term on the left-hand side of this equation. This is a natural consequence of the assumption that the memory kernel must tend to zero with a negative tail as an inverse power law. With this assumption it is straightforward to prove that the modulus of the first term becomes much larger than that of the second term on the left-hand side of this equation. The consequences of this crucial assumption are supported by the numerical results depicted in Fig. 1. With this assumption Eq. (32) simplifies to

$$
-2 \int_{0}^{t} \Phi_{a}\left(t-t^{\prime}\right) \frac{1}{t^{\prime \beta}} d t^{\prime}=\frac{\beta t}{(t+1)^{\beta+1}},
$$

which can be solved by means of the fractional calculus [2]. We use the Riemann-Liouville (RL) definition of the fractional integral:

$$
D_{t}^{-q}[f(t)]=\frac{1}{\Gamma(q)} \int_{0}^{t} \frac{f\left(t^{\prime}\right) d t^{\prime}}{\left(t-t^{\prime}\right)^{1-q}},
$$

which is the antiderivative of the fractional derivative with order $q$, with $q<1$. Consequently, for $\beta<1$ we can express Eq. (33) in terms of the RL fractional integral

$$
D_{t}^{\beta-1}\left[\Phi_{a}(t)\right]=-\frac{1}{2 \Gamma(1-\beta)} \frac{\beta t}{(t+1)^{\beta+1}},
$$

so that inverting this equation we have the formal expression for the slow part of the memory kernel

$$
\begin{aligned}
\Phi_{a}(t) & =-\frac{1}{2 \Gamma(1-\beta)} D_{t}^{1-\beta}\left[\frac{\beta t}{(t+1)^{\beta+1}}\right] \\
& =-\frac{1}{2 \Gamma(1-\beta)} D_{t}^{1-\beta}\left[\beta t \Phi_{\xi, \beta+1}(t)\right] .
\end{aligned}
$$

We denote the autocorrelation function with the inverse power $(\beta+1)$ by the symbol $\Phi_{\xi, \beta+1}(t)$. Carrying out the required calculations on the right-hand side of Eq. (35), we obtain (see, for example, West et al. [[2], p. 90], 


$$
\Phi_{a}(t)=-\frac{\beta}{2 \Gamma(1-\beta) \Gamma(1+\beta)} \frac{t^{\beta}}{(t+1)^{2}}, \quad \beta<1 .
$$

A comparison with numerical inversion of the kernel is shown in Fig. 1.

For the sake of completeness, it is worth noticing that we can proceed in a similar way in the case $\beta>1$ also. In this case we find, for the contribution $\Phi_{a}(t)$ of the GME, the following time asymptotic behavior:

$$
\Phi_{a}(t)=-\frac{\beta(\beta-1)}{2} \frac{t}{(t+1)^{\beta+1}}, \quad \beta>1 .
$$

\section{THE EMERGENCE OF FRACTIONAL OPERATORS}

In this section we show that in the two-site case we are discussing the GME has the form of a transport equation, with two terms on the right-hand side. The first has the form afforded by the ordinary master equation and consequently satisfies the Onsager principle, giving a relaxation dependent on the average waiting time of the CTRW. The second term corresponds to a fractional derivative in time, and extends the Onsager principle to the case of a relaxation with a fat tail. To obtain these results, we use what we have learned in the preceding section.

First of all, since we are dealing with the two-site case, using the form of $\mathbf{M}$ and $\mathbf{K}$ matrices with $z=x=-1$ and $y$ $=1$, we rewrite Eq. (1) in the following form:

$$
\begin{aligned}
& \frac{d}{d t} p_{1}(t)=\int_{0}^{t} \Phi(t-\tau)\left[p_{2}(\tau)-p_{1}(\tau)\right] d \tau, \\
& \frac{d}{d t} p_{2}(t)=\int_{0}^{t} \Phi(t-\tau)\left(p_{1}(\tau)-p_{2}(\tau)\right) d \tau .
\end{aligned}
$$

The memory kernel $\Phi$ is related to the autocorrelation function of the dichotomous variable $\Phi_{\xi}$ through Eq. (17). Inserting Eq. (17) into the Laplace transform of the set of the two-site dynamical equations, solving the resulting set of equations, and taking the corresponding inverse Laplace transforms yields the solutions

$$
\begin{aligned}
& p_{1}(t)=\frac{1}{2}\left\{1-\Phi_{\xi}(t)\left[p_{2}(0)-p_{1}(0)\right]\right\}, \\
& p_{2}(t)=\frac{1}{2}\left[1+\Phi_{\xi}(t)\left(p_{2}(0)-p_{1}(0)\right)\right] .
\end{aligned}
$$

Note that these solutions can be combined to yield the generalized Onsager principle given by Eq. (10) in terms of the difference in the probabilities.

We now want to find a formal equation of evolution for the probabilities involving fractional operators. We know from the preceding section that

$$
\Phi(t)=\frac{\delta(t)}{2 \tau^{*}}+\Phi_{a}(t)
$$

with $\tau^{*}=T^{*} /(\mu-2)=1 / \beta$, thanks to the fact that we set $T^{*}$ $=1$. Substituting the decomposition of the memory kernel into Eq. (38), we obtain

$$
\frac{d p_{1}(t)}{d t}=\frac{p_{2}(t)-p_{1}(t)}{2 \tau^{*}}+\int_{0}^{t} \Phi_{a}(t-\tau)\left[p_{2}(\tau)-p_{1}(\tau)\right] d \tau .
$$

Writing $\Phi_{a}(t)$ as the derivative of an as yet unspecified function $f(t)$, and using the property

$$
\frac{d}{d t} \int_{0}^{t} f(t-\tau) g(\tau) d \tau=f(0) g(t)+\int_{0}^{t} f^{\prime}(t-\tau) g(\tau) d \tau
$$

with $f^{\prime}(t)=(d / d t) f(t)$, we obtain

$$
\begin{aligned}
\frac{d p_{1}(t)}{d t}= & \frac{p_{2}(t)-p_{1}(t)}{2 \tau^{*}}-f(0)\left[p_{2}(t)-p_{1}(t)\right]+\frac{d}{d t} \int_{0}^{t} f(t-\tau) \\
& \times\left[p_{2}(\tau)-p_{1}(\tau)\right] d \tau .
\end{aligned}
$$

We also found that in the case $0<\beta<1$ the asymptotic behavior of the memory kernel is expressed by

$$
\begin{aligned}
\Phi_{a}(t) & \approx-\frac{\beta}{2 \Gamma(1-\beta) \Gamma(1+\beta)} \frac{t^{\beta}}{(t+1)^{2}} \\
& \approx-\frac{\beta}{2 \Gamma(1-\beta) \Gamma(1+\beta)} t^{\beta-2}, \quad t \rightarrow \infty, \quad T^{*}=1 .
\end{aligned}
$$

Rewriting Eq. (47) as

$$
\Phi_{a}(t) \approx-\frac{1}{2 \Gamma(1-\beta) \Gamma(\beta)(\beta-1)} \frac{d}{d t} t^{\beta-1},
$$

we identify $f(t)$ with $[1 / 2(1-\beta) \Gamma(1-\beta) \Gamma(\beta)] t^{\beta-1}$ for $t \rightarrow \infty$. Choosing $f(0)=0$ and using the properties of the $\Gamma$ function, we assign to the time asymptotic equation of motion the form

$$
\begin{aligned}
\frac{d p_{1}(t)}{d t}= & -\frac{p_{1}(t)-p_{2}(t)}{2 \tau^{*}}+\frac{1}{2 \Gamma(2-\beta) \Gamma(\beta)} \frac{d}{d t} \int_{0}^{t}(t \\
& -\tau)^{\beta-1}\left[p_{2}(\tau)-p_{1}(\tau)\right] d \tau
\end{aligned}
$$

or, in terms of the RL fractional integral (34),

$$
\frac{d p_{1}(t)}{d t}=-\frac{p_{1}(t)-p_{2}(t)}{2 \tau^{*}}-\frac{1}{2 \Gamma(2-\beta)} D_{t}^{1-\beta}\left[p_{1}(t)-p_{2}(t)\right] .
$$

The same procedure applied to the equation of motion for $p_{2}(t)$ yields

$$
\frac{d p_{2}(t)}{d t}=-\frac{p_{2}(t)-p_{1}(t)}{2 \tau^{*}}+\frac{1}{2 \Gamma(2-\beta)} D_{t}^{1-\beta}\left[p_{1}(t)-p_{2}(t)\right] .
$$


The difference between these two equations yields

$$
\begin{aligned}
\frac{d}{d t}\left[p_{1}(t)-p_{2}(t)\right]= & -\frac{p_{1}(t)-p_{2}(t)}{\tau^{*}}-\frac{1}{\Gamma(2-\beta)} D_{t}^{1-\beta}\left[p_{1}(t)\right. \\
& \left.-p_{2}(t)\right],
\end{aligned}
$$

clearly showing the two kinds of contribution to the generalized Onsager principle. The first term gives the relaxation of the perturbation away from equilibrium at the macroscopic rate required by Onsager. The second term gives the additional slow relaxation in the form of the fractional integral.

\section{AGING ORDER}

We have to remark that the condition of Eq. (12) refers to the stationary condition explicitly considered by Klafter and Zumofen [29]. In this section we prove that there is a connection between a system's age and the order of the fractional derivative in the relaxation process. A sign of the dependence of the fractional derivative order on age is given by the discrepancy between the results of Sec. III and Ref. [12]. Let us compare Eq. (49) to Eq. (16) of Ref. [12]. We obtain the fractional index $1-\beta$ rather than $\beta$ as in the work of Sokolov and Metzler. In Appendix A we prove that our time asymptotic approach to fractional derivatives, in the nonstationary case studied by Sokolov and Metzler, yields the same index as they obtain [12]. Thus, the discrepancy between our prediction and the prediction of Sokolov and Metzler depends on the fact that we consider a condition consistent with the Onsager principle, whereas Sokolov and Metzler do not. Furthermore, if the system is not infinitely aged, a sort of rejuvenation process is expected to take place that will lead to the fractional order of Sokolov and Metzler.

To support our remarks concerning the relation between aging and the order of the fractional operator, here we discuss how to define a waiting time distribution of any age. The authors of Ref. [13] have shown that the waiting time distribution $\psi(t)$ of Eq. (14) is obtained from the following dynamic model. A particle moves in an the interval $I$ $\equiv[0,1]$ driven by the equation of motion

$$
d y / d t=\alpha y^{z},
$$

with $z>1$. When the particle reaches the border $y=1$, it is injected back to an initial condition between $y=0$ and $y=1$ with uniform probability. The age of the CTRW is determined by the distribution of first exit times. The ordinary CTRW is based on identifying this distribution with $\psi(t)$. This means that the CTRW is equivalent to assuming that the system is prepared in a flat distribution at $t=0$, which coincides with the beginning of the observation process.

Let us discuss now the consequence of beginning the observation a significant time after the preparation. Let us imagine that the system is prepared in a flat distribution at a time $t=-t_{a}<0$, and that the observation begins at $t=0$. This means that the flat distribution begins producing a sequence of time intervals of size $\tau$, according to the distribution of Eq. (14); more precisely, the time interval $\tau_{1}$ beginning at $t$ $=-t_{a}$ and ending at $t=-t_{a}+\tau_{1}$, the time interval $\tau_{2}$ beginning at $t=-t_{a}+\tau_{1}$ and ending at $t=-t_{a}+\tau_{1}+\tau_{2}$, and so on. The waiting time distribution of age $t_{a}$, denoted by $\psi_{t_{a}}(t)$, is determined by the first of these time intervals overlapping with $t>0$. The time length of that overlap is the time length whose distribution determines $\psi_{t}(t)$. We make the assumption that the beginning of the first time interval overlapping with $t>0$ occurs with equal probability at any point between $t=-t_{a}$ and $t=0$. The validity of this assumption is discussed in Appendix B, which establishes that this assumption is very good for $t_{a} \rightarrow 0$ and $t_{a} \rightarrow \infty$. In between the asymptotic limits the resulting prediction is not exact. However, since it yields simple analytical formulas, we adopt this simplifying assumption for any age. Thus, we have that

$$
\psi_{t_{a}}(t)=\frac{\int_{0}^{t_{a}} \psi(t+y) d y}{g\left(t_{a}\right)},
$$

where $g\left(t_{a}\right)$ is the normalization factor defined by

$$
g\left(t_{a}\right) \equiv \int_{0}^{t_{a}} \Psi\left(t^{\prime}\right) d t^{\prime},
$$

and $\Psi(t)$ is the probability that no event occurs throughout the time interval of length $t$. Using for $\psi(t)$, according to the prescription adopted in this paper, the analytical form of Eq. (26), it is easy to prove that Eq. (53) can be written in the form

$$
\psi_{t_{a}}(t)=(\mu-2) \frac{(t+T)^{(1-\mu)}-\left(t+T+t_{a}\right)^{(1-\mu)}}{T^{(2-\mu)}-\left(t_{a}+T\right)^{(2-\mu)}} .
$$

This formula proves that for $t \ll t_{a}$ the index of the distribution is $\mu-1$, whereas for $t \gg t_{a}$ the index becomes $\mu$. This result for the age-dependent waiting time distribution function agrees with the predictions by Barkai [26] and by the authors of Ref. [13]. Notice that the formula Eq. (55) is equivalent to drawing the initial condition for $y$ from an aged distribution of this variable.

Here, we are in a position to evaluate the waiting time index at a generic time, where we write $\psi_{t_{a}}(t)$ as

$$
\psi_{t_{a}}(t)=\frac{A\left(T, t_{a}\right)}{(t+T)^{\mu_{\mathrm{eff}}(t)}}
$$

Using Eq. (55) we arrive at the following formula for the time dependence of the effective power-law index:

$$
\mu_{\mathrm{eff}}(t)=\frac{\ln \left[(t+T)^{(1-\mu)}-\left(t+T+t_{a}\right)^{(1-\mu)}\right]}{\ln [t+T]} .
$$

Figure 2 illustrates the regression of the effective powerlaw index to $\mu$ with two different ages, and shows clearly that the regression is slower for older systems. This formula does more than explain the discrepancy between Eq. (49) and Eq. (16) of Ref. [12]. In fact, it shows that it is possible to build a GME that at short times follows the prescription of our GME and at long times moves into the basin of attraction of Sokolov and Metzler. This is certainly the case if $t_{a}>-\infty$. 


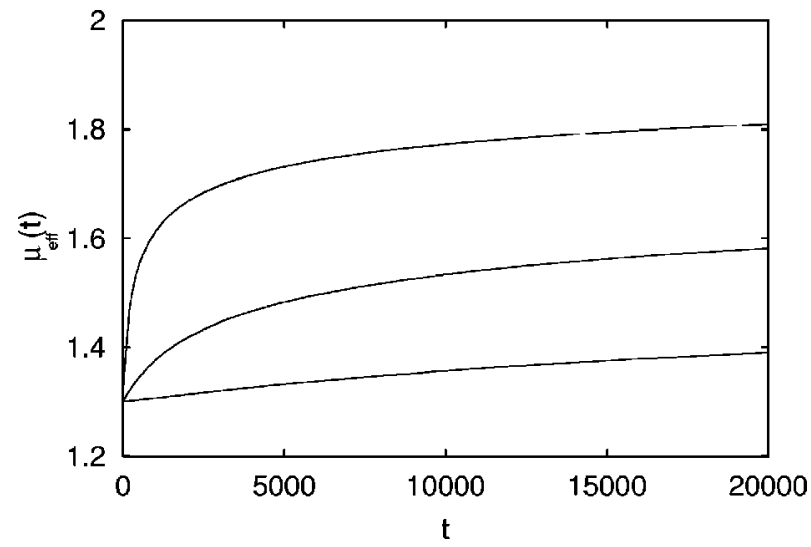

FIG. 2. The effective power index $\mu_{\mathrm{eff}}(t)$ as a function of time, for $\mu=2.3$. The curves refer, from top to bottom, to $t_{a}=100,1000$, 10000 .

This aspect is important and needs a more exhaustive illustration. We note that the approach of Ref. [13] can be easily extended to the case where the distribution of first exit times has a finite age. It is enough to follow the procedure of Ref. [13] and to replace the first exit time distribution with $\psi_{t_{a}}(t)$ rather than with $\psi_{\infty}(t) \equiv \psi_{t_{a}=-\infty}(t)$, as done in Ref. [13]. The result of this procedure yields for the GME the following form for the Laplace transform of the memory kernel:

$$
\hat{\Phi}_{t_{a}}(u)=\frac{u \hat{\psi}_{t_{a}}(u)}{1+\hat{\psi}(u)-2 \hat{\psi}_{t_{a}}(u)} .
$$

It is straightforward to prove that for $t_{a}=0 \mathrm{Eq}$. (58) reduces to

$$
\hat{\Phi}_{t_{a}=0}(u)=\frac{u \hat{\psi}(u)}{1-\hat{\psi}(u)} .
$$

In fact, the general prescription of Eq. (53) immediately yields $\psi_{t_{a}}(t)=\psi(t)$ for $t_{a}=0$. To derive the memory kernel corresponding to the infinitely aged condition of Eq. (15) we have to notice first that Eq. (53) yields, in accordance with Refs. [26] and [13],

$$
\psi_{\infty}(t) \equiv \psi_{t_{a}=\infty}(t)=\frac{1}{\tau} \int_{t}^{\infty} d t^{\prime} \psi\left(t^{\prime}\right) .
$$

To illustrate the change of the memory kernel with time, notice that the Laplace transform of Eq. (55) is

$$
\hat{\psi}_{t_{a}}(u)=\frac{\hat{\Psi}(u)\left(1-e^{u t_{a}}\right)+e^{u t_{a}} \int_{0}^{t_{a}} e^{-u y} \Psi(y) d y}{g\left(t_{a}\right)},
$$

that is,

$$
\hat{\psi}_{t_{a}}(u)=\frac{[1-\hat{\psi}(u)]\left(1-e^{-u t_{a}}\right)+u e^{u t_{a}} \int_{0}^{t_{a}} e^{-u y} \Psi(y) d y}{u g\left(t_{a}\right)} .
$$

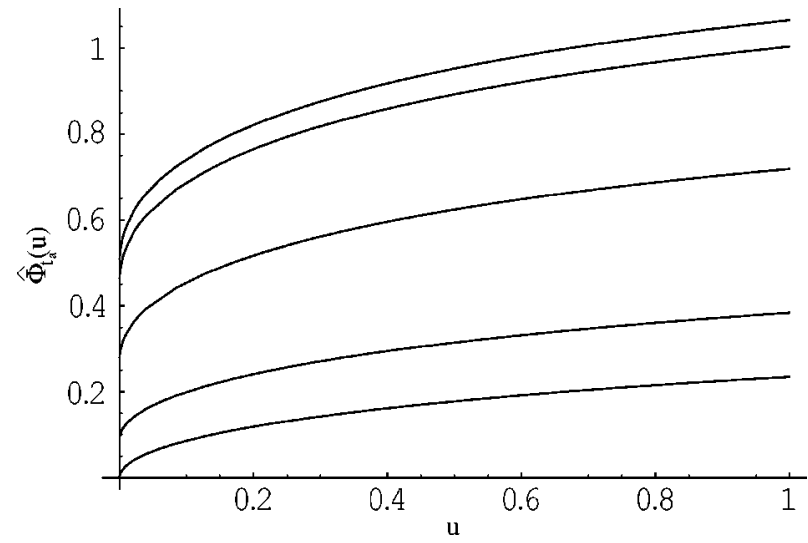

FIG. 3. The Laplace transform of the $t_{a}$-old memory kernel $\Phi_{t_{a}}(t), \hat{\Phi}_{t_{a}}(u)$, as a function of $u$, for $\mu=2.3$. The curves refer, from top to bottom, to $t_{a}=0,0.1,1,10, \infty$.

Let us substitute Eq. (62) into Eq. (58), from which we obtain the Laplace transform of the memory kernel for an arbitrary age. In Fig. 3 we show the Laplace transform of the memory kernel corresponding to a number of different ages. Moving from the top to the bottom curve the age increases from the brand new kernel $\left(t_{a}=0\right)$ to the infinitely aged or stationary kernel $\left(t_{a}=\infty\right)$.

\section{CONCLUDING REMARKS}

The adoption of a two-state model to generate anomalous diffusion is not unusual in the random walk literature. For instance, Shushin [30] also generated anomalous diffusion by means of a two-state model. However, to properly locate in the literature of anomalous diffusion the model of the present paper, we have to point out that the diffusion generator in Ref. [30] is a two-state Markov equation modulated by timedependent transition parameters that obey non-Poisson statistics. The model studied in the present paper might be adopted to generate anomalous diffusion as well, but is quite different from that of Sushin. The extended time of sojourn in one of the two states would produce uniform motion, with no randomness involved, as discussed by the authors of Ref. [12], which rests in fact on the same model as that adopted here.

In this paper we establish that an infinitely old system, with the power-law index in the interval $2 \leqslant \mu \leqslant 3$, yields the fractional order $1-\beta=3-\mu$. The prediction of Ref. [12], on the other hand, yields the fractional order $\mu-2$, corresponding to the brand new condition. If the system is not infinitely aged, namely, $t_{a}<\infty$, the short-time behavior of the system for $t \ll t_{a}$ is expected to be that of an aged system. At large observation times, $t \gg t_{a}$, rejuvenation begins. This can be explained using the dynamical model of Eq. (52). In fact, aging has to do with the slow regression to equilibrium, if it exists, of the variable $y$, which mimics a real bath slowly regressing to equilibrium. The aging effects discussed by Barkai [26] correspond to $z>2$ and thus to $\mu<2$. In this case the dynamic model under discussion does not have an invariant distribution, and, consequently, any observation is 
done while the bath is drifting toward a condition that will never be reached. This aging effect affects the form of the first exit time distribution, whose index is $\mu-1$ rather than $\mu$. However, after the first exit the trajectories are injected back with a uniform probability, and thus all the ensuing jumps are determined by the ordinary waiting time distribution $\psi(t)$.

It would be desirable to have an equation of motion with a fractional operator order that changes as a function of time from $3-\mu$ to $\mu-2$. However, there are technical and conceptual difficulties that make it difficult, if not impossible, to realize this goal. In fact, according to the perspective adopted in this paper, the order of the fractional operator is established using time asymptotic arguments. Thus, if $t_{a}<\infty$ we can associate a time $t \ll t_{a}$ with the order $a=\mu-2$ of the fractional derivative. This is so because for $t \ll t_{a}$ the calculations would be virtually equivalent to those done in Appendix A. It is not clear how to proceed when $t$ is of the order of $t_{a}$, this being the first reason why assigning a fractional derivative order to any time might be difficult. There also exists a physical reason that might make it impossible to move from the order $3-\mu$ to $\mu-2$. Physically, this extremely extended transition process might involve a mixture of fractional derivatives of different orders. It has been shown [31] that the Lévy walk does not have well-defined scaling, due to aging effects. Similarly, the adoption of a fractional time derivative, with time-dependent order, might be inadequate to explore the regime of transition from the order $3-\mu$ to the order $\mu-2$. In conclusion, the fractional operator and its order reflect a stable condition, of a brand new or infinitely old system. The regime of transition from the dynamic to any of these two thermodynamic regimes, and the regime of transition from the earlier to the latter thermodynamic regime, is not yet a fully understood physical condition, an issue calling for further investigation.

It is interesting to notice that, even if we select $\mu>2$ and consequently adopt a condition compatible with the stationary condition, the effective index of the first exit distribution is located in the nonstationary region if $\beta<1$. This is probably the reason why the memory kernel seems to share the same properties as those adopted in Refs. $[6,10,32]$ to produce subdiffusion. Notice that the baths used by Lutz [6] and Pottier [32] have properties quite different from the subordination perspective of Ref. [10], even though the relaxation process stemming from subordination [10] is quite similar to that produced by the non-Ohmic baths of Lutz and Pottier. We hope that the present work might help in understanding the connection between the two perspectives. This is another subject for future research.

\section{ACKNOWLEDGMENTS}

G. A. and P. G. gratefully acknowledge financial support from ARO through Grant No. DAAD190210037.

\section{APPENDIX A}

In this appendix we show how to obtain the order of the fractional operator in the GME for $1<\mu<3$, using the Laplace transform form for $\Phi(t)$ given by Eq. (8). We are using $\psi(t)$ rather than $\psi^{*}(t)$. Thus, according to the procedure adopted in this paper, we use the analytical form of Eq. (26). For the reader's convenience, we rewrite this expression here,

$$
\psi(t)=(\mu-1) \frac{T^{\mu-1}}{(T+t)^{\mu-1}} .
$$

It is important to stress that in the text we made the choice of Eq. (20) for $\psi^{*}(t)$, in this way determining, through Eq. (24), a form for $\psi(t)$ that departs from Eq. (A1). However, both choices lead to the same time asymptotic behavior of Eq. (14) for both the waiting time distributions.

We note that for $u \rightarrow \infty$ we get a finite value $\hat{\Phi}(\infty)=(\mu$ $-1) / T$ corresponding to $\psi(0)$ in $t$ space. As in Sec. II, we separate the kernel into two contributions: $\Phi(t)=[(\mu$ $-1) / T] \delta(t)+\Phi_{a}(t)$, and insert the Laplace transform of separation into Eq. (8), to write

$$
\begin{aligned}
\hat{\Phi}_{a}(u) & =\frac{u \hat{\psi}(u)-(\mu-1)+(\mu-1) \hat{\psi}(u)}{1-\hat{\psi}(u)} \\
& =\frac{\hat{\psi}_{D}(u)+(\mu-1) \hat{\psi}(u)}{1-\hat{\psi}(u)} .
\end{aligned}
$$

We introduce $\hat{\psi}_{D}(u)$ as the Laplace transform of the distribution's derivative $\psi^{\prime}(t)=-\mu(\mu-1) T^{\mu-1} /(T+t)^{\mu+1}$; using the Laplace transform of an inverse power law and substituting it into Eq. (A2) we have

$$
\begin{aligned}
\hat{\Phi}_{a}(u)= & \frac{(\mu-1)^{2} \Gamma(1-\mu)\left(e^{u}-E_{\mu-1}^{u}\right)}{u^{1-\mu}-(\mu-1) \Gamma(1-\mu)\left(e^{u}-E_{\mu-1}^{u}\right)} \\
& -\frac{\mu(\mu-1) \Gamma(-\mu) u\left(e^{u}-E_{\mu}^{u}\right)}{u^{1-\mu}-(\mu-1) \Gamma(1-\mu)\left(e^{u}-E_{\mu-1}^{u}\right)},
\end{aligned}
$$

where, as usual, for simplicity we have set $T=1$. Anticipating the convolution form of the solution we cross-multiply to obtain

$$
\begin{gathered}
{\left[u^{1-\mu}-(\mu-1) \Gamma(1-\mu)\left(e^{u}-E_{\mu-1}^{u}\right)\right] \hat{\Phi}_{a}(u)=(\mu-1)[(\mu} \\
\left.-1) \Gamma(1-\mu)\left(e^{u}-E_{\mu-1}^{u}\right)\right]-\mu \Gamma(-\mu) u\left(e^{u}-E_{\mu}^{u}\right) .
\end{gathered}
$$

Using the relation [2]

$$
\int_{0}^{\infty} \frac{t^{\alpha}}{t+a} \exp [-u t] d t=\frac{\pi a^{\alpha}}{\sin \pi \alpha}\left(E_{\alpha}^{u a}-e^{u a}\right), \quad \alpha>-1,
$$

and setting $\alpha=\mu-1$, we construct

$$
\begin{aligned}
R(t)= & \frac{1}{\Gamma(\mu-1)} \int_{0}^{t} t^{\prime \mu-2} \Phi_{a}\left(t-t^{\prime}\right) d t^{\prime}-\frac{\sin \pi \mu}{\pi}(\mu-1) \Gamma(1 \\
& -\mu) \int_{0}^{t} \frac{t^{\prime \mu-1}}{t^{\prime}+1} \Phi_{a}\left(t-t^{\prime}\right) d t^{\prime},
\end{aligned}
$$

where $R(t)$ is the inverse Laplace transform of the right side of Eq. (A4). Using the well known recurrence relation of the $\Gamma$ function, we can combine terms in Eq. (A6) to obtain 


$$
\begin{aligned}
R(t) & =\frac{1}{\Gamma(\mu-1)} \int_{0}^{t} t^{\prime \mu-2}\left(1-\frac{t^{\prime}}{t^{\prime}+1}\right) \Phi_{a}\left(t-t^{\prime}\right) d t^{\prime} \\
& =\frac{1}{\Gamma(\mu-1)} \int_{0}^{t} \frac{t^{\prime \mu-2}}{t^{\prime}+1} \Phi_{a}\left(t-t^{\prime}\right) d t^{\prime} .
\end{aligned}
$$

Let us consider first the case $\mu>2$, where, in the limit of $t$ $\gg 1$ (equivalent to $t \gg T$ ), we can write Eq. (A7) to a good approximation as

$$
R(t)=\frac{1}{\Gamma(\mu-1)} \int_{0}^{t} t^{\prime \mu-3} \Phi_{a}\left(t-t^{\prime}\right) d t^{\prime}=R(t) .
$$

Going back to the Laplace transform representation, we obtain the simplier expression

$$
\begin{aligned}
\frac{\Gamma(\mu-2)}{\Gamma(\mu-1)} \frac{\hat{\Phi}_{a}(u)}{u^{\mu-2}}= & (\mu-1)^{2} \Gamma(1-\mu)\left(e^{u}-E_{\mu-1}^{u}\right) \\
& -\mu(\mu-1) \Gamma(-\mu) u\left(e^{u}-E_{\mu}^{u}\right),
\end{aligned}
$$

which after a little algebra yields for $\hat{\Phi}_{a}(u)$

$$
\begin{aligned}
\hat{\Phi}_{a}(u)= & (\mu-1)(\mu-2)\left[(\mu-1) \Gamma(1-\mu) \frac{1}{u} \frac{e^{u}-E_{\mu-1}^{u}}{u^{1-\mu}}\right. \\
& \left.-\mu \Gamma(-\mu) \frac{1}{u} \frac{\left(e^{u}-E_{\mu}^{u}\right)}{u^{-\mu}}\right] .
\end{aligned}
$$

Thus using the inverse Laplace transforms we obtain the corresponding expression in the time representation:

$$
\Phi_{a}(t)=(\mu-1)(\mu-2)\left[\frac{1}{(1+t)^{\mu}}-\frac{1}{(1+t)^{\mu-1}}\right] .
$$

In the time asymptotic limit we get

$$
\Phi_{a}(t) \propto \frac{1}{t^{\mu-1}},
$$

corresponding to a fractional operator of index $\beta=\mu-2$. For the sake of completeness, we also give the expression for the GME kernel in the case $\mu<2$. Proceeding as was done earlier we obtain

$$
\Phi(t) \approx-\frac{\sin \pi \mu}{\pi} \frac{t^{\mu-2}}{(t+1)^{2}}[\mu-1+(\mu-2) t] .
$$

Finally, we want to point out that the expressions we are proposing refer to values of $\mu$ which are not integer. We are exploring the interval $[1,3]$. Thus the the expressions we are proposing become questionable for $\mu=2$. To get the proper expression for $\mu=2$ we have to study expressions like those of Eq. (A2) and (A9) at $\mu=2+\epsilon$, do a Taylor series expansion around $\mu=2$, and assign to $\mu=2$ the limiting values reached for $\epsilon \rightarrow 0$.

\section{APPENDIX B}

This appendix is devoted to establishing the accuracy of Eq. (53), and consequently the validity of the assumption

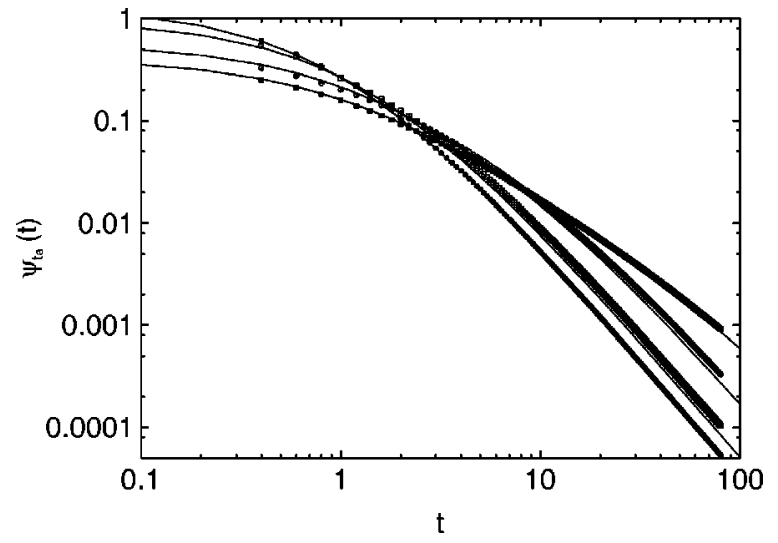

FIG. 4. The waiting time distribution $\psi_{t_{a}}(t)$ as a function ot time $t$. The dots denote the exact values, and the lines the prediction of Eq. (55) for $\mu=2.3, T=1$. Moving from bottom to top in the righthand portion of the figure, $t_{a}=0.01,1,10,100$.

that the beginning of the first laminar region overlapping with $t>0$ is uniformly distributed between $t=-t_{a}$ and $t=0$.

The exact expression for $\psi_{t_{a}}(t)$ is

$$
\psi_{t_{a}}(t)=\int_{0}^{t_{a}} d x G\left(t_{a}-x\right) \psi(t+x),
$$

where

$$
\begin{aligned}
G(t) \equiv & \delta(t)+\psi(t)+\sum_{n=2}^{\infty} \int_{0}^{t} d \tau_{1} \psi\left(\tau_{1}\right) \cdot \int_{\tau_{1}}^{t} d \tau_{2} \psi\left(\tau_{2}\right. \\
& \left.-\tau_{1}\right) \cdots \int_{\tau_{n-2}}^{t} d \tau_{n-1} \psi\left(t-\tau_{n-1}\right) .
\end{aligned}
$$

It is straightforward to find the Laplace transform of $G(t)$. This is given by

$$
\hat{G}(u)=\sum_{n=0}^{\infty} \hat{\psi}(u)^{n}=\frac{1}{1-\hat{\psi}(u)} .
$$

Thus, the Laplace transform of Eq. (B1) with respect to $t_{a}$ reads

$$
\psi_{s_{a}}(t)=\frac{1}{1-\psi\left(s_{a}\right)} e^{s_{a}}\left[\psi\left(s_{a}\right)-\int_{0}^{t} e^{-s_{a} y} \psi(y) d y\right] .
$$

By numerically anti-Laplace transforming Eq. (B4) we evaluate the time dependence of the exact waiting time distribution of age $t_{a}$, Eq. (B1).

In Fig. 4 we compare the exact prediction, evaluated numerically, to the heuristic expression of Eq. (53). We find that at small and large values of $t_{a}$ these two expressions coincide. In the intermediate region they do not. Nevertheless, we think the agreement between the two expressions is satisfactory enough for the purpose of this paper. 
[1] Applications of Fractional Calculus in Physics, edited by R. Hilfer (World Scientific, Singapore, 2000).

[2] B. J. West, M. Bologna, and P. Grigolini, Physics of Fractal Operators (Springer, New York, 2003).

[3] R. Metzler and J. Klafter, Phys. Rep. 339, 1 (2000).

[4] G. M. Zaslavsky, Phys. Rep. 371, 461 (2002).

[5] R. Metzler and A. Compte, J. Phys. Chem. B 104, 3858 (2000).

[6] E. Lutz, Phys. Rev. E 64, 051106 (2001).

[7] A. V. Chechkin, R. Gorenflo, and I. M. Sokolov, Phys. Rev. E 66, 046129 (2002).

[8] J. Bisquert, Phys. Rev. Lett. 91, 010602 (2003).

[9] R. Metzler and J. Klafter, J. Phys. Chem. B 1043851 (2000).

[10] E. Barkai and R. J. Silbey, J. Phys. Chem. B 1043866 (2000).

[11] A. V. Chechkin, J. Klafter, V. Yu. Gonchar, R. Metzler, and L. V. Tanatarov, Phys. Rev. E 67, 010102(R) (2003).

[12] I. M. Sokolov and R. Metzler, Phys. Rev. E 67, 010101(R) (2003).

[13] P. Allegrini, G. Aquino, P. Grigolini, L. Palatella, and A. Rosa, Phys. Rev. E 68, 056123 (2003).

[14] L. Onsager, Phys. Rev. 38, 2265 (1931); 37, 405 (1931).

[15] R. Kubo, Can. J. Phys. 34, 1274 (1956).

[16] E. W. Montroll and G. H. Weiss, J. Math. Phys. 6, 167 (1965).

[17] D. Bedeaux, K. Lakatos, and K. Shuler, J. Math. Phys. 12, 22116 (1971).
[18] V. M. Kenkre, E. W. Montroll, and M. F. Shlesinger, J. Stat. Phys. 9, 45 (1973).

[19] R. Metzler Phys. Rev. E 62, 6233 (2000).

[20] E. W. Montroll and B. J. West, in Fluctuation Phenomena, edited by E. W. Montroll and J. L. Lebowitz (North-Holland, Amsterdam, 1979).

[21] T. Geisel, J. Nierwetberg, and A. Zacherl, Phys. Rev. Lett. 54, 616 (1985).

[22] F. Bardou, J.-P. Bouchaud, A. Aspect, and C. CohenTannoudji, Lévy Statistics and Laser Cooling (Cambridge University Press, Cambridge, England, 2002).

[23] X. Brokmann, J.-P. Hermier, G. Messin, P. Desbiollles, J.-P. Bouchaud, and M. Dahan, Phys. Rev. Lett. 90, 120601 (2003).

[24] I. M. Sokolov, A. Blumen and J. Klafter, Europhys. Lett. 56, 175 (2001).

[25] G. Zumofen and J. Klafter, Phys. Rev. E 47, 851 (1993).

[26] E. Barkai, Phys. Rev. Lett. 90, 104101 (2003).

[27] M. Bologna, P. Grigolini, and B. J. West, Chem. Phys. 284, 115 (2002).

[28] A. Fuliński, Phys. Rev. E 50, 2668 (1994).

[29] J. Klafter and G. Zumofen, Physica A 196, 102 (1993).

[30] A. I. Shushin, Phys. Rev. E 64, 051108 (2001).

[31] P. Allegrini, J. Bellazzini, G. Bramanti, M. Ignaccolo, P. Grigolini, and J. Yang, Phys. Rev. E 66, 015101R (2002).

[32] N. Pottier, Physica A 317, 371 (2003). 